\title{
Electronic structure of self-assembled quantum dots: comparison between density functional theory and diffusion quantum Monte Carlo
}

\author{
J. Shumway ${ }^{a, *, \dagger}$, L. R. C. Fonseca ${ }^{b, \ddagger)}$, J. P. Leburton ${ }^{b, c)}, \operatorname{Richard~M.~Martin~}^{a, b)}$, D. M. Ceperley ${ }^{a, b)}$ \\ a) Department of Physics \\ ${ }^{b)}$ Beckman Institute for Advanced Science and Technology \\ ${ }^{c)}$ Department of Electrical and Computer Engineering \\ University of Illinois at Urbana-Champaign, Urbana, IL 61801
}

(October 29, 2018)

\begin{abstract}
We have calculated the exchange, correlation, and total electronic energy of a realistic InAs selfassembled quantum dot embedded in a GaAs matrix as a function of the number of electrons in the dot. The many-body interactions have been treated using the local spin density approximation (LSDA) to density functional theory (DFT) and diffusion quantum Monte Carlo (DMC), so that we may quantify the error introduced by LSDA. The comparison shows that the LSDA errors are about 1-2 meV per electron for the system considered. These errors are small enough to justify the use of LSDA calculations to test models of self-assembled dots against current experimental measurements.
\end{abstract}

PACS Numbers: $85.30 . \mathrm{Vw}$

\section{INTRODUCTION}

The electronic structure of quantum dots resembles that of atoms. Both systems display three dimensional electronic confinement leading to level degeneracies, shell structure, and spin correlation to only mention the most studied atomic properties of quantum dots. 10 Direct as well as exchange Coulomb interactions are also present in quantum dot systems, the importance of each depending on the dot dimensions. That is why some of the theoretical methods used to calculate the electronic structure of quantum dots are the same as the ones used to study atoms and molecules. One of these methods, local spin density approximation (LSDA) density functional theory (DFT) within the effective mass approximation (EMA), has been widely used to calculate the electron-electron interaction in dots 11 because of its simple implementation and low computer demand. LSDA is an approximate theory, well known to predict incorrectly the physical properties of some molecules and solids, while performing well on many other systems. 12 However, experience with LSDA on atoms and molecules may not carry over to quantum dots since the confining potentials and electron densities can be very different. As a general rule, density functional theory tends to work better for high effective density, and fails for low density systems, where correlation effects become important. There have been several investigations of LSDA and exact treatment of electron interactions in parabolic quantum dots, 13,16 with particular emphasis on low density systems and effects of external magnetic fields. The electron interactions in parabolic dots have been treated exactly using diffusion Monte Carlo(DMC) 13, 4 as well as exact diagonalization methods 15.16 . The parabolic potentials are popular models because they are computationally convenient and, with two adjustable parameters, have had relative success in explaining the influence of many-body effects on the electronic properties of dots 6
In this paper we compare EMA results obtained with LSDA in self-assembled InAs/GaAs quantum dots against an exact treatment of the EMA many-body inter actions obtained with diffusion quantum Monte Carlo.17 These small dots, which can hold about six electrons, have complicated confining potentials that differ considerably from the parabolic dots considered in previous DMC and exact diagonalization calculations. The single particle states of realistically modeled pyramidal dots are significantly modified from the states in a parabolic dot 1 so many-body interactions should also differ from those in parabolic models. The purpose of this comparison is to quantify errors due to LSDA for calculations on realistically modeled InAs/GaAs dots. Although error of LSDA is expected to be small in these systems, it is large enough to be a limitation to the comparison of model results to experiments. For the system reported here, we consider LSDA errors of up to $10 \mathrm{meV}$ to be acceptable given the current precision of the models and experiments. Determination of whether LSDA meets this acceptability criterion requires careful comparison with many-body calculations on realistically modeled potentials.

In these calculations we consider the ground state energy as a function of the number of electrons in the dot, which is the quantity for which DFT should apply. Electron addition and removal energies are experimentally measurable. These may be rigorously defined as differences in the total energies of the ground states of the dot which differ by one electron. A common approach to calculating electron addition and removal energies is to use the eigenvalues of the DFT equations, which are well known not to be interpretable as electron addition and removal enrrgies. We have addressed this issue in a previous papent where we have shown that addition and removal energies are to a very gegd approximation given by the "Slater transition rule", 18 which uses the eigenvalues at one-half occupation. Thus the present work, 
which compares total energies, is a rigorous test of the LSDA, and combined with our previous work is a test of the accuracy of the addition and removal energies obtained using LSDA eigenvalues and the Slater transition rules.

The ground state energy is the lowest eigenvalue of the many-body Schrödinger equation, which we take to be
The total energy can then written as

$$
E=E_{\mathrm{kin}}+E_{\mathrm{cb}}[\rho]+E_{\mathrm{H}}[\rho]+E_{\mathrm{x}}[\rho]+E_{\mathrm{c}}[\rho],
$$

where

$$
E_{\mathrm{kin}}=-\frac{\hbar^{2}}{2} \sum_{\sigma, i=1}^{N} \int \psi_{i}^{*}(\mathbf{r}) \nabla\left(M^{-1} \nabla\right) \psi_{i}(\mathbf{r}, \sigma) d \mathbf{r}
$$

$$
\begin{gathered}
\left\{-\sum_{i=1}^{N} \frac{\hbar^{2}}{2} \nabla_{i}\left[M^{-1} \nabla_{i}\right]+V_{\mathrm{cb}}(R)+V_{\mathrm{ee}}(R)\right\} \Psi(R)=E \Psi(R), \text { is the kinetic energy of the Kohn-Sham orbitals, } \\
E_{\mathrm{cb}}[\rho]=\int \rho(\mathbf{r}) v_{\mathrm{cb}}(\mathbf{r}) d \mathbf{r},
\end{gathered}
$$

where $\Psi(R)=\Psi\left(\mathbf{r}_{1}, \mathbf{r}_{2}, \cdots \mathbf{r}_{N}\right)$ is the $N$-electron wave function, $M$ is the electron effective mass tensor and the potential energy terms $V_{\mathrm{cb}}(R)$ and $V_{\mathrm{ee}}(R)$ are given by

$$
\begin{aligned}
& V_{\mathrm{cb}}(R)=\sum_{i=1}^{N} v_{\mathrm{cb}}\left(\mathbf{r}_{i}\right) \\
& V_{\mathrm{ee}}(R)=\sum_{i<j} \frac{e^{2}}{\epsilon\left|\mathbf{r}_{i}-\mathbf{r}_{j}\right|}
\end{aligned}
$$

where $v_{\mathrm{cb}}(\mathbf{r})$ is the potential for a single electron due to the offset and strain potential of the conduction band, and $V_{\mathrm{ee}}(R)$ is the Coulomb interaction of the conduction electrons with charge $-e$ in a dielectric characterized by the dielectric constant $\epsilon$. The offset of the conduction band edge causes a step potential at the edge of the dot, which is reduced by strain, resulting in a potential $v_{\mathrm{cb}}$ that is zero outside the dot and a roughly constant depth of several tenths of an $\mathrm{eV}$ in the interior of the dot, which typically has dimensions $\approx 10 \mathrm{~nm}$. Strain causes the electron effective mass to become anisotropic leading to a mass tensor given by $\operatorname{diag}(M)=\left(m_{\mathrm{xx}} m_{\mathrm{yy}} m_{\mathrm{zz}}\right)$ and zero off-diagonal terms. In the usual case of sample growth along the crystal direction $\left(\begin{array}{lll}0 & 0 & 1\end{array}\right)$, the electron masses along the plane perpendicular to the growth direction are equal, i.e. $m_{\mathrm{xx}}=m_{\mathrm{yy}}$.

\section{DENSITY FUNCTION THEORY AND THE LOCAL SPIN DENSITY APPROXIMATION}

The DFT approach to obtaining the ground state energy is to replace the rather complex N-electron ground state wave function and the associated Schrödinger equation by the much simpler ground state electron density $\rho(\mathbf{r})$ and the corresponding functional forms $T[\rho]$ and $V[\rho]$ of the ginetic and potential energy operators, respectively. 1921 However, those functional forms are unknown, and mpproximations are necessary. In KohnSham theory 22 the density is given by a sum of densities of single particle orbitals,

$$
\rho(\mathbf{r})=\sum_{\sigma} \sum_{i=1}^{N} n_{i}\left|\psi_{i}(\mathbf{r}, \sigma)\right|^{2} .
$$

is the potential energy from the conduction band offset and strain,

$$
E_{\mathrm{H}}[\rho]=-e \int \rho(\mathbf{r}) \phi(\mathbf{r}) d \mathbf{r},
$$

is the Hartree energy, and $E_{\mathrm{x}}[\rho]$ and $E_{\mathrm{c}}[\rho]$ are functionals of the electron density. The self-consistent electrostatic potential $\phi(\mathbf{r})$ is obtained as a solution to the Poisson equation

$$
\nabla^{2} \phi(\mathbf{r})=\frac{e}{\epsilon} \rho(\mathbf{r})
$$

with the boundary condition $\phi(\mathbf{r}) \rightarrow 0$ as $|\mathbf{r}| \rightarrow \infty$. Minimization of the total energy defined by Eqs. (19-9) yields a one particle Schrödinger equation which may be solved self-consistently with the expression for the density and the Poisson equation to determine the exact many-body ground state energy. However, the nonlocal functionals $E_{\mathrm{x}}[\rho]$ and $E_{\mathrm{c}}[\rho]$ are in general not known, so an approximation must be made in order to proceed with the KohnSham approach.

In the local spin density approximation (LSDA) the exchange and correlation functionals are taken to be funcuniquely defined by the requirement that the approximation be exact for the homogeneous electron gas. These functions have been fit to the exchange and correlation of the uniform electron gas.23 The validity of LSDA has been a subject of much research in atomic, crystallipe molecular, and parabolic quantum dot systems,20.21, 13 but has not been checked for self-assembled dot potentials, which are qualitatively like finite square wells with rather high electron density. The density gradients and shell structures of self-assembled dots cause the exchange and correlation energy to differ from that of a uniform gas. The purpose of this paper is to quantify this difference as a function of the number of electrons in a realistically modeled dot.

\section{QUANTUM MONTE CARLO METHODS}

The simplest QMC method is variational Monte Carlo (VMC), in which expectation values for a trial wave function are evaluated using a Metropolis algorithm. Using tions of the local charge and spin density, which are then 
the variational principle of the ground state energy, parameters in the wave function can be optimized to minimize the total energy, thus providing an approximation to the ground state energy and wave function. In our calculation we use VMC to optimize the guiding wave function for our diffusion Monte Carlo algorithm, described below.

We also use Monte Carlo integration to evaluate the exact exchange energy. In the Kohn-Sham formalism, the exchange energy is defined as the difference

$$
E_{\mathrm{x}}[n]=\left\langle\Psi_{\mathrm{KS}}\left|V_{\mathrm{ee}}\right| \Psi_{\mathrm{KS}}\right\rangle-E_{\mathrm{H}}[\rho],
$$

where $\left|\Psi_{\mathrm{KS}}\right\rangle$ is a Slater determinant of the true KohnSham orbitals; those that give the exact density. We assume that LSDA is accurate enough to provide approximations to the Kohn-Sham orbitals, $i$. $e$. that the LSDA density is close to the exact density. We then evaluate the integral $\left\langle\Psi_{\mathrm{KS}}\left|V_{\mathrm{ee}}\right| \Psi_{\mathrm{KS}}\right\rangle$ using a Slater determinant of our calculated LSDA wave functions.

Diffusion Monte Carlo is a stochastic method that is able to project the N-electron ground state wave function $\Phi_{0}$ from a trial wave function $\Psi_{\mathrm{T}}$. A position basis is used to described the state of the system. A good trial wave function is important because the variance of the Monte Carlo sampling decreases as the trial wave function approaches the true ground state wave function. We chose the trial wave function to be a product of Slater determinants for spin up and spin down electrons with a Jastrow factor that introduces correlation,

$$
\Psi_{\mathrm{T}}(R)=\operatorname{det}\left[\theta_{\mathrm{k}}\left(\mathbf{r}_{\mathrm{i}}\right)\right] \times \exp \left[-\sum_{\mathrm{i}<\mathrm{j}} u\left(r_{\mathrm{ij}}\right)\right] .
$$

For convenience, we take the single particle wave functions $\theta_{\mathrm{k}}(\mathbf{r})$ to be the non-interacting eigenstates for the external potential, and choose the Jastrow factor $u(r)=$ $-a r /(1+b r)$ with $a=m / \epsilon$ and $b=1 / 2$.

Following the method described in Ref. [ 17], we used importance sampling from a function $\Phi(R)=$ $\Psi_{\mathrm{T}}(R) \Phi_{0}(R)$. The anisotropic mass is taken into account using the mass tensor, $M$. The projection of a state $R$ to the ground state distribution $\Psi_{\mathrm{T}} \Phi_{0}$ is accomplished by repeatedly applying the projection operator $e^{-H \tau}$, each time sampling a new configuration. Here $H$ is the Hamiltonian and $\tau$ is a parameter chosen to be small so that the projection operator is approximated by 17

$$
\begin{aligned}
\left\langle R\left|\Psi_{\mathrm{T}}(R) e^{-H \tau} \Psi_{\mathrm{T}}^{-1}\left(R^{\prime}\right)\right| R^{\prime}\right\rangle & \\
= & \frac{\exp \left\{-\left[E_{\mathrm{L}}(R)-E_{0}\right] \tau\right\}}{\left[(2 \pi \tau)^{3} \operatorname{det} M^{-1}\right]^{\frac{n}{2}}} \times \\
& \prod_{i=1}^{n} \exp \left[-\frac{\left(\mathbf{r}_{\mathrm{i}}-\mathbf{r}_{\mathrm{i}}-\tau \mathbf{v}_{\mathrm{d}, \mathrm{i}}\right) \cdot M \cdot\left(\mathbf{r}_{\mathrm{i}}-\mathbf{r}_{\mathrm{i}}-\tau \mathbf{v}_{\mathrm{d}, \mathrm{i}}\right)}{2 \tau}\right],
\end{aligned}
$$

where $E_{0}$ is a constant parameter to control the population, $E_{\mathrm{L}}(R)=\Psi_{\mathrm{T}}(R)^{-1} H \Psi_{\mathrm{T}}(R)$ is the local energy, and $\mathbf{v}_{\mathrm{d}}=M^{-1} \cdot \nabla \log \Psi_{\mathrm{T}}$. The algorithm is thus: (1) Start with an ensemble of configurations (walkers) distributed

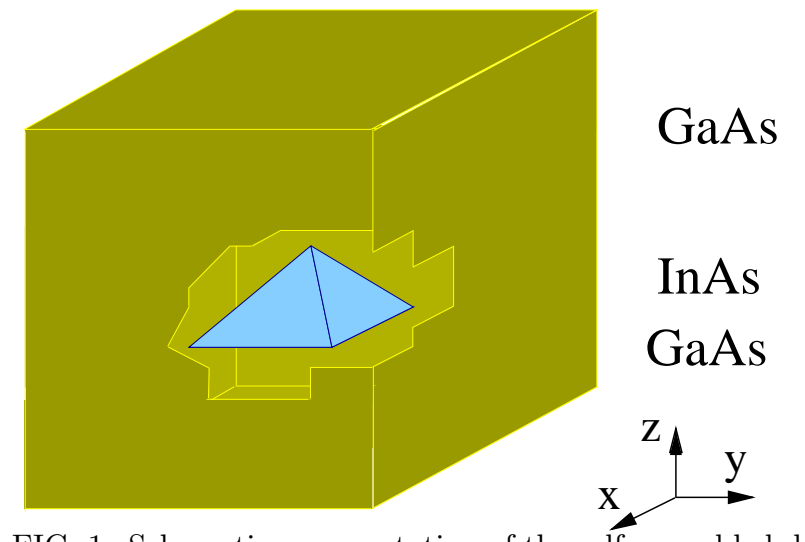

FIG. 1. Schematic representation of the self-assembled dot structure used in the present work.

by $\left|\Psi_{\mathrm{T}}\right|^{2},(2)$ propagate each configuration with a drift $\mathbf{v}_{\mathrm{d}}$ and Gaussian displacement with covariance matrix $\sigma_{\mathrm{ij}}^{2}=M_{\mathrm{ij}}^{-1} \tau,(3)$ reweight each configuration by a factor $\exp \left\{-\left(E_{\mathrm{L}}-E_{0}\right) \tau\right\}$, (4) repeat, collecting statistics once the steady state distribution is reached. The exact ground state energy is obtained by sampling the local energy. We use branching 17 at each step to improve the efficiency of the process.

A complication known as the fermion sign problem arises when applying diffusion Monte Carlo to fermions. Electron exchange introduces negative signs into the projection operator, which decreases the efficiency of the Monte Carlo sampling. In the present discussion the short time approximation, Eq. (12), breaks down when walkers cross nodes of $\Psi_{\mathrm{T}}$. We handle this problem with the fixed node approximation, 24 in which walkers are given a weight of zero when they cross a node of $\Psi_{\mathrm{T}}$. This approximation has a lower bound property, so the true ground state energy can only be lower than the fixed node energy, and the exact choice of $\Psi_{\mathrm{T}}=\Phi_{0}$ would give the exact Fermionic ground state energy.

The correlation energy can be deduced from a knowledge of all other energies,

$$
E_{c}[\rho]=E_{\mathrm{tot}}-E_{\mathrm{kin}}-E_{\mathrm{cb}}-E_{\mathrm{H}}-E_{\mathrm{x}},
$$

or equivalently

$$
E_{c}[\rho]=\left\langle\Phi_{0}\left|V_{\mathrm{ee}}\right| \Psi_{0}\right\rangle-\left\langle\Psi_{\mathrm{KS}}\left|V_{\mathrm{ee}}\right| \Psi_{\mathrm{KS}}\right\rangle .
$$

\section{NUMERICAL COMPARISON}

Using DFT and DMC we have calculated the total ground state energy of the system of electrons, defined for DFT by Eq. 5, and defined within DMC as the fixed node ground state energy, as defined in the previous section. Figure 1 shows the system we have considered, a pyramidal shaped InAs quantum dot in a GaAs matrix. However, our method of analysis is not restricted to this particular shape, and we expect our results to be valid 
for truncated pyramidal or lens shaped dots. We have introduced several simplifications in the model. Since our DMC scheme does not allow for a position dependent dielectric constant, we have used only one value, $\bar{\epsilon}=14 \epsilon_{0}$, for both GaAs and for InAs. In the absence of strain the GaAs - InAs conduction band offset between the two materials is $770 \mathrm{meV}$. To partially take into account strain effects, we have assumed a constant shift of $400 \mathrm{meV}$ in the InAs conduction band, and no strain effect in the GaAs conduction band, resulting in a conduction band offset of $770 \mathrm{meV}-400 \mathrm{meV}=370 \mathrm{meV}$. We also take the electron effective masses to be constant in each material.

In order to study a realistic potential, we have used a nonuniform grid basis for the LSDA calculation and the solution of the Poisson equation. For the Schrödinger equation we set the wave functions to zero at the edge of the grid, which is reasonable due to the exponential decay of bound states, but the Poisson equation requires more care. We used a multipole expansion up to the quadrupole term to simulate the boundary conditions at infinity. This difficulty with the boundary conditions of the Poisson equation is one source of error in our comparison. We used cubic interpolation to map the gridded wave functions and conduction band potential to continuous functions for Monte Carlo evaluation of the exchange energy, for determination of the trial nodes in the DMC calculation, and for guiding wave functions in the DMC calculation. Although the cubic interpolation of the potential allows us to compare LSDA and DMC for similar external potentials $v_{\mathrm{cb}}$, errors in the kinetic energy operator are more difficult. The use of a finite difference approximation to the Laplacian in the computation of the LSDA solution creates an operator that has no simple counterpart in a continuous formulation of the problem. The error between the physical Laplacian operator and the artificial finite difference operator can be made arbitrarily small by the use of finer grids, but computer memory and CPU time constraints caused this to be a significant source of error in our comparison. The size of the errors are several $\mathrm{meV}$, which are comparable to the rather small LSDA errors, especially in the correlation energy. Below we discuss a way we estimated this error so that we can compare correlation energy.

For the set of calculations we describe now, we have taken all masses to have the isotropic value of $0.05 m_{e}$, for both the InAs and GaAs regions. The case of anisotropic masses will be discussed later. We have also accounted for numerical discrepancies between the two calculations due to grid interpolation error. To estimate this error we have compared eigenvalues of single electron states with both methods, and found that the DMC single particle eigenvalues typically lie about $0.25 \mathrm{meV}$ above the eigenvalues computed by the grid method used in the DFT calculation. We attribute that difference to a systematic error in the finite difference kinetic energy operator and shift up our LSDA calculations to compensate for this.

Figure 2 shows the values of the different components of the total energy as a function of dot occupation. Dif-

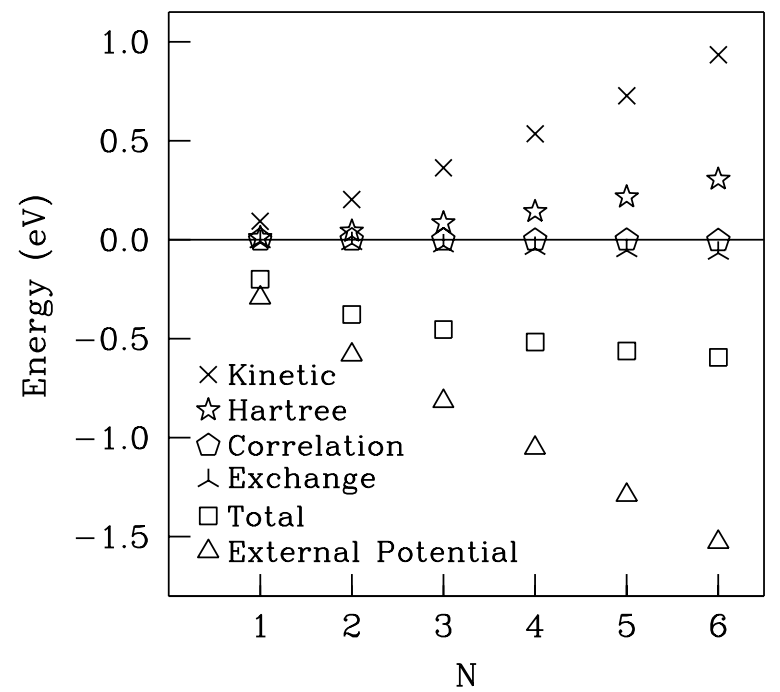

FIG. 2. Contributions to the energy of a $200 \times 100 \AA$ pyramidal dot as a function of occupation $N$, calculated by both LSDA and QMC. Differences between LSDA and QMC are not apparent on the energy scale of this figure. This clearly shows the small effect of interactions beyond the Hartree (mean field) level.

ferences between LSDA and QMC are not apparent on the scale of the figure. This figure clearly shows that the external potential energy and kinetic energy are much larger than the interaction energies. In other words the effects of interactions enter as a perturbative correction to the non-interacting system. The reason for this can be seen from the scaled electron density. The energy and length scales for the electron interaction are scaled by the dielectric constant and mass, so that the effective Bohr radius is $a_{0}^{*}=\epsilon / m^{*} a_{0} \approx 150 \AA$ and the effective Hartree is $\mathrm{Ha}^{*}=\epsilon^{-2} m^{*} \mathrm{Ha} \approx 7 \mathrm{meV}$. If we estimate the density by assuming the $N$ electrons uniformly occupy the interior of the dot, we find an effective conduction electron density of $r_{s} \approx 0.46 N^{-1 / 3}$. To see the consequences of such a high effective density, consider the uniform electron gas at $r_{s}=0.6$. The electron gas has a ground state energy expansion for small $r_{s}, 25$

$$
E=2.2099 r_{s}^{-2}-0.9163 r_{s}^{-1}-0.094+0.0622 \ln \left(r_{s}\right)+\cdots,
$$

where the first term is the kinetic energy, the next term is the exchange, and remaining terms are correlation energy. For the case of six electrons in the $\operatorname{dot} r_{s} \approx 0.25$ and the expansion gives $E_{\text {kin }} \approx 1440 \mathrm{meV}, E_{\mathrm{x}} \approx 150 \mathrm{meV}$, and $E_{\mathrm{c}} \approx 0.6 \mathrm{meV}$. Although the comparison between these very different electronic systems cannot be pushed too far, this does show that our exchange and correlation energies are reasonable for this electron density. The leading effect of the interactions is the Hartree energy, with small corrections for exchange and very small correlation 


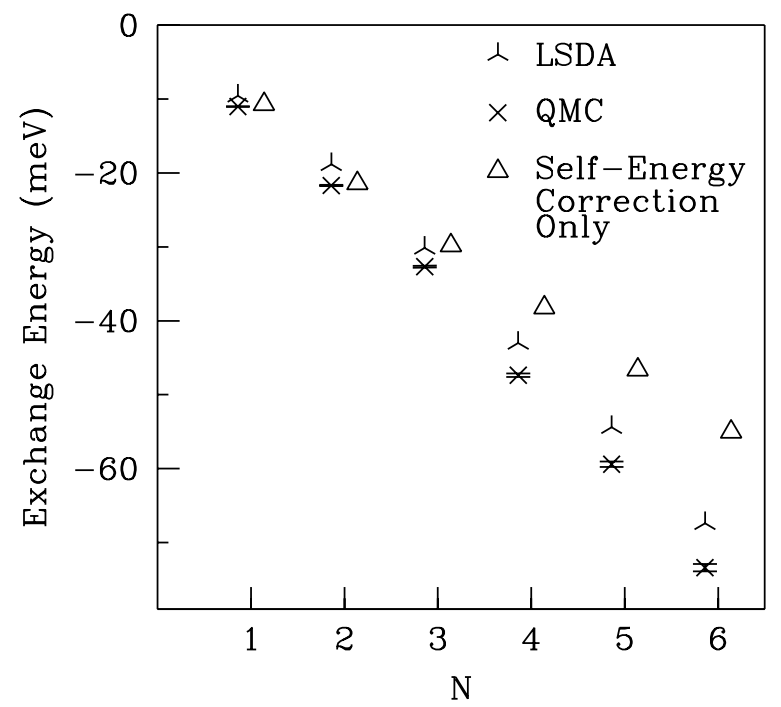

FIG. 3. Exchange energy of a $200 \times 100 \AA$ pyramidal dot as a function of occupation $N$, as determined by LSDA and QMC. Also shown is the self-energy correction, which makes a large contribution to the exchange energy.

corrections.

The most important contribution of LSDA in this system is the exchange term, and we plot the comparison of the LSDA exchange energy in Figure 3. For finite systems, the definition for exchange includes the correction for self-interaction in the Hartree energy 20 which we also show in the figure. Merely correcting for self-interaction will recover at least $75 \%$ of the exchange energy, and LSDA is able to do better, recovering about $90 \%$ for an error in the exchange energy of less than $5 \mathrm{meV}$. As is well known in many other systems 20 , the error in the local approximation to the exchange is the largest error in LSDA.

By comparison the correlation energy is much smaller and its accuracy is difficult to assess due to grid errors. Correlation is defined as the difference in total energies, Eq. (14), and in this case the total energy difference is less than $1 \%$. Thus our estimates of the exact correlation energy are fairly uncertain. In Figure 1 we show our best attempt at checking the LSDA correlation in the quantum dot. Since the LSDA wave functions are not the true Kohn-Sham orbitals (due mostly to grid errors) the correlation is overestimated due to relaxation of our interpolated LSDA states. As stated earlier, we correct for this by performing single particle calculations to estimate the relaxation of our LSDA states, and have removed this contribution from our calculated correlation energy. These results are shown as "QMC" and "QMC with corrections" in the figure. We find that LSDA gives a reasonable estimate of the magnitude of the correlation energy in this system.

Our comparison of the energy errors from the LSDA are summarized in Figure 5. The largest error is due to

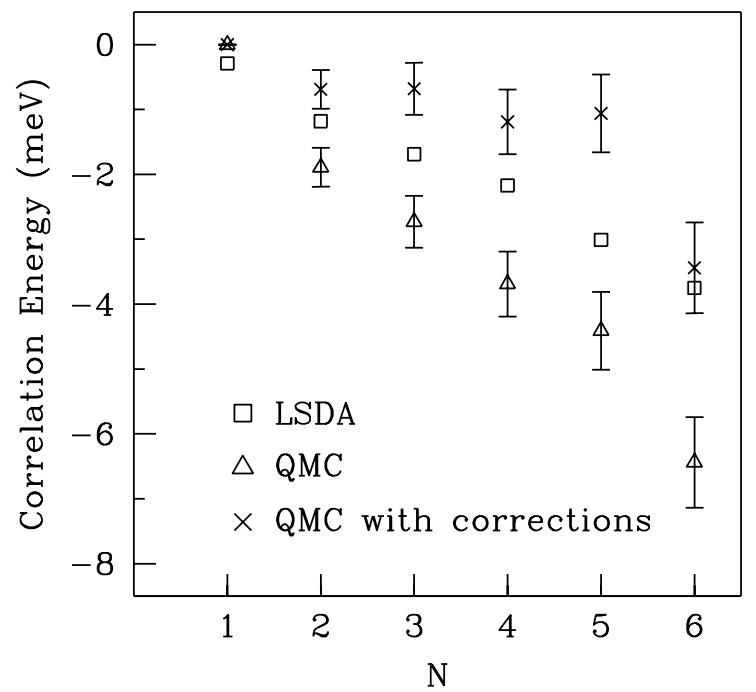

FIG. 4. Correlation energy of a $200 \times 100 \AA$ pyramidal dot as a function of occupation $N$, as determined by LSDA, QMC, and QMC with corrections for grid interpolation error, as explained in the text. LSDA gives a reasonable approximation to this small quantity.

LSDA underestimating the magnitude of the exchange energy, $E_{\mathrm{x}}$, which is a $6 \mathrm{meV}$ error for six electrons in the dot. There is a small error in the correlation energy, $E_{\mathrm{c}}$, which slightly compensates for the error in the exchange. The error in the Hartree energy $E_{\mathrm{Ha}}$ is due primarily to truncation of the multipole expansion at quadrupole terms for determination of the boundary conditions of the Poison equation. There are also errors in $E_{\text {kin }}$ and $E_{\mathrm{cb}}$ (not shown in the figure) due to the grid, in particular the discretization of the kinetic energy operator discussed earlier. We therefore find that LSDA leads to an error in the total energy of up to $6 \mathrm{meV}$, which in our calculation is partially compensated for by grid errors in other energy terms, leading to an overall error in the total energy of no more than $2 \mathrm{meV}$.

Because of strain, the electron effective mass in the plane parallel to the base of the det is usually different from the mass perpendicular to it. The LSDA does not explicitly account for mass anisotropy in the exchange and correlation functionals, thus for the calculation of the exchange-correlation potential $V_{\mathrm{xc}}$ we have assumed a constant average mass given by

$$
m_{\mathrm{xc}}=\left(m_{\|}^{2} m_{\perp}\right)^{1 / 3},
$$

where $m_{\|}$is the in-plane mass, $m_{\|}=m_{\mathrm{xx}}=m_{\mathrm{yy}}$, and $m_{\perp}$ is the perpendicular mass, $m_{\perp}=m_{\mathrm{zz}}$. The choice of the average mass $m_{\mathrm{xc}}$ is somewhat arbitrary. We have performed a test of this approximation by calculating the exchange and correlation energy for an anisotropic system using (i) LSDA with $m_{\mathrm{xc}}$ as given above, (ii) LSDA with $m_{\mathrm{xc}}$ optically averaged, $m_{\mathrm{xc}}^{-1}=\left(2 / m_{\|}+1 / m_{\perp}\right) / 3$, and (iii) DMC, which can explicitly treat anisotropic 


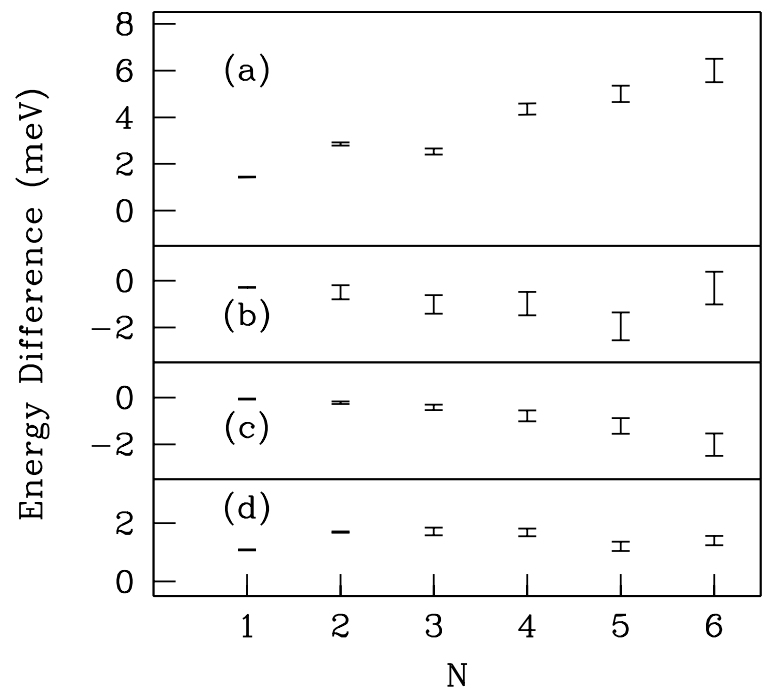

FIG. 5. Difference in energies between LSDA and QMC $\left(E_{\mathrm{LSDA}}-E_{\mathrm{DMC}}\right)$ for a $200 \times 100 \AA$ pyramidal dot as a function of occupation $N$ : (a) error in the exchange energy, $E_{\mathrm{x}}$, due mostly to LSDA, (b) error in correlation energy, $E_{\mathrm{c}}$, due to grid interpolation error and LSDA, with some grid corrections as described in the text, (c) error in Hartree energy $E_{\mathrm{Ha}}$, due to grid interpolation error and the multipole expansion, and (d) error in the total energy. The values in (a), (b) and (c) do not add up to the value in (d) due to errors in $E_{\mathrm{cb}}$ and $E_{\text {kin }}$ (not shown).

mass. Figure 6 shows the calculated exchange and correlation energies for several electron mass ratios, which we have again fixed to constant across the InAs and GaAs. We find that either choice is acceptable, as both give errors comparable to the isotropic mass case.

\section{CONCLUSIONS}

In conclusion, we have shown that DFT offers an accurate approximation for the ground state energy including many-body interactions in small self-assembled quantum dots, while providing a simple and fast means of modeling systems containing several electrons. This is in large part due to the small size of the self-assembled quantum dots in relation to the effective Bohr radius. In this regime the largest error is in the local approximation to the exchange, and we have verified that the errors due to this approximation are small. This is quantified in Figures 2 and 3 where we see that the exchange energy for six electrons in the dot is $72 \mathrm{meV}$ with an LSDA error of $6 \mathrm{meV}$, compared to a total energy of $1490 \mathrm{meV}$. We expect this applicability of LSDA to hold in general for small quantum dots of various shapes, but we emphasize that LSDA is expected to become progressively worse if the dot becomes much larger than the effective Bohr radius. Also, the use of the EMA Schrödinger equation,

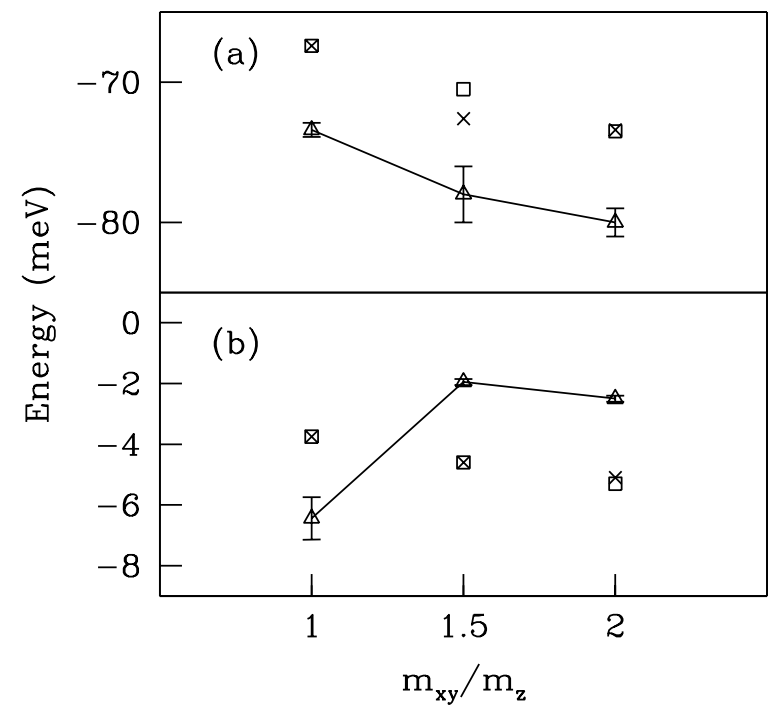

FIG. 6. Values of the (a) exchange and (b) correlation energy as a function of mass anisotropy for six electrons in a $200 \times \AA$ pyramid dot. Triangles are DMC results, $\times$ 's are LSDA results with $m_{\mathrm{xc}}^{-1}=\left(2 m_{\|}^{-1}+m_{\perp}^{-1}\right) / 3$, and squares are LSDA results with $m_{\mathrm{xc}}=\left(m_{\|}^{2} * m_{\perp}\right)^{1 / 3}$.

Eq. (11), although reasonable for the chosen problem, is predicted to fail for very small dots.26 Although our model assumes pure InAs dots surrounded by GaAs, in some systems indium and gallium may in fact intermix.27 Again, we expect the relative magnetude of exchange and correlation energies and the acceptability of LSDA to be unchanged by intermixing or alloying of the dots.

Based on this comparison we conclude that the addition and removal energies found by EMA-LSDA calculations in realistic dots are accurate to $1-2 \mathrm{meV}$ per electron as far as the many-body interactions within the EMA are concerned. Thus, comparison of such calculated charging energies with experiment can be considered a direct test of the models and the uncertainties in the analysis of experiments. carefully tested the grid errors and concluded that energies are accurate to $\approx \pm 10 \mathrm{meV}$ including all errors.

We would like to point out that application of LSDA to a system of several coupled self-assembled dots may have difficulties. Systems that have weak intersite transitions along with intrasite interactions are a well-known manybody problem where LSDA is known to fail. The problem is closely related to the $\mathrm{H}_{2}$ molecule, where LSDA describes well the electronic energy at equilibrium distance, but gives an incorrect broken symmetry solution if the atoms are pulled apart beyond a critical distance. The problem of weakly coupled dots would be of particular importance for future studies. 


\section{ACKNOWLEDGMENTS}

This work was supported by CRI from the University of Illinois and NSF Grants No. ECS 95-09751 and No. DMR 94-22496 and computer resources at NCSA.

* Electronic address: shumway@uiuc.edu

$\dagger$ Present address: National Renewable Energy Laboratory, Golden, CO 80401

¥ Present address: Motorola Flat Panel Display Division, Tempe, AZ 85284

${ }^{1}$ M. Fricke, A. Lorke, J. P. Kotthaus, G. Medeiros-Ribeiro, and P. M. Petroff, Europhys. Lett. 36, 197 (1996).

${ }^{2}$ S. Tarucha, D. G. Austing, T. Honda, R. J. van der Hage, and L. P. Kouwenhoven, Phys. Rev. Lett 77, 3613 (1996).

${ }^{3}$ H. Drexler, D. Leonard, W. Hansen, J. P. Kotthaus, and P. M. Petroff, Phys. Rev. Lett. 73, 2252 (1994).

${ }^{4}$ J. -Y. Marzin, J. -M. Gérard, A. Izraël, and D. Barrier, G. Bastard, Phys. Rev. Lett. 73, 716 (1994).

${ }^{5}$ M. Grundman, J. Christen, N. N. Ledentsov, J. Böhrer, D. Bimberg, S. S. Ruvimov, P. Werner, U. Richter, U. Gösele, J. Heydenreich, V. m. Ustinov, A. Yu. Egorov, A. E. Zhukov, P. S. Kop'ev, and Zh. I. Alferov, Phys. Rev. Lett. 74, 4043 (1995).

${ }^{6}$ A. Wojs and P. Hawrylak, Phys. Rev. B 53, 10841 (1996).

${ }^{7}$ L. R. C. Fonseca, J. L. Jimenez, J. P. Leburton, and Richard M. Martin, Phys. Rev. B 57, 4017 (1998), and erratum Phys. Rev. B 58, 1691 (1998).

${ }^{8}$ S. Nagaraja, P. Matagne, V. Y. Thean, J. P. Leburton, Y. H. Kim, and R. M. Martin, Phys. Rev. B 56, 15752 (1997).

${ }^{9}$ I.-H. Lee, V. Rao, R. M. Martin, and J. P. Leburton, Phys. Rev. B 57, 9035 (1998).

${ }^{10}$ M. Macucci, Karl Hess, and G. J. Iafrate, Phys. Rev. B 55, R4879 (1997).

${ }^{11}$ M. Koskinen, M. Manninen, and S. M. Reimann, Phys. Rev. Lett. 79, 1389 (1997).

${ }^{12}$ R. O. Jones, in Ab Initio Methods in Quantum Chemistry, edited by K. P. Lawley (Wiley, New York, 1987), p. 413.

${ }^{13}$ C. Filippi, C. J. Umrigar, and M. Taut, J. Chem. Phys. 100, 1290 (1994).

${ }^{14}$ F. Bolton, Phys. Rev. B 54, 4780 (1996).

${ }^{15}$ S.-R. Eric Yang, A. H. MacDonald, and M. D. Johnson, Phys. Rev. Lett. 71, 3194 (1993).

${ }^{16}$ M. Eto, Jpn. J. Appl. Phys. 36, 3924 (1997).

${ }^{17}$ D. M. Ceperley and M. H. Kalos, in Monte Carlo Methods in Condensed Matter Physics, edited by K. Binder, Topics in Current Physics, Vol. 7 (Springer, Heidelberg, 1979), Chap. 4; K. E. Schmidt and M. H. Kalos, in Applicatoins of the Monte Carlo Methods in Condensed Matter Physics, edited by K. Binder, Topics in Current Physics, Vol. 36 (Springer, Heidelberg, 1984), Chap. 4;

18 J. C. Slater, Adv. Quantum Chem. 6, 1 (1972).

19 P. Hohenberg and W. Kohn, Phys. Rev. 136, B864 (1964).

${ }^{20}$ A more complete description of DFT can be found in R. G.
Parr and W. Yang, Density-Functional Theory of Atoms and Molecules, (Oxford University Press, New York, 1989).

${ }^{21}$ R. M. Dreizler and E. K. U. Gross, Density Functional Theory: An Approach to the Quantum Many-body Problem, (Springer, Berlin, 1990).

22 W. Kohn and L. J. Sham, Phys. Rev. 140, A1133 (1976).

23 J. P. Perdew and Y. Wang, Phys. Rev. B 45, 13244 (1992).

${ }^{24}$ P. J. Reynolds, D. M. Ceperley, B. J. Alder, and W. A. Lester, J. Chem. Phys. 77, 5593 (1982).

${ }^{25}$ G. D. Mahan Many-Particle Physics, (Plenum, New York, 1990).

${ }^{26}$ A. Franceschetti and Alex Zunger, Phys. Rev. Lett. 78, 915 (1997).

27 S. Skolnick, et al., to be published in Physica E, Proc of the EP2DS-XIII, Ottawa, Canada, 1999. 